\title{
Hemorragia submembrana limitante interna em paciente após Valsalva: relato de caso
}

\section{Sub-internal limiting membrane hemorrhage in Valsalva retinopathy: case report}

\author{
Marcelo Mendes Lavezzo $^{1}$, Leandro Cabral Zacharias $^{1}$, Walter Yukihiko Takahashi ${ }^{2}$
}

\section{RESUMO}

Relatar o caso de uma paciente com retinopatia por Valsalva que evoluiu com hemorragia submembrana limitante interna e foi submetida à vitrectomia via pars plana, tendo tido melhora da acuidade visual. Paciente hígida de 35 anos evoluiu com redução súbita e indolor da acuidade visual do olho direito, após crise de tosse. Ao exame oftalmológico, apresentava hemorragia pré-macular, sem outras alterações. Inicialmente, optou-se pela conduta expectante, porém não houve regressão completa da hemorragia. Então, foi indicada a vitrectomia via pars plana associada à remoção da membrana limitante interna, havendo melhora considerável da acuidade visual, sem complicações peri-operatórias e sem alterações significativas à tomografia de coerência óptica, autofluorescência ou eletrorretinograma multifocal. Neste caso, o tratamento da hemorragia submembrana limitante interna com a cirurgia vitreorretiniana resultou em melhora da acuidade visual e regressão da hemorragia.

Descritores: Hemorragia retiniana; Manobra de Valsalva; Vitrectomia; Tomografia de coerência óptica; Acuidade visual; Humanos; Feminino; Adulto; Relatos de caso

\begin{abstract}
To report a case of a patient with Valsalva retinopathy that developed sub-internal limiting membrane hemorrhage, underwent pars plana vitrectomy and had visual acuity improvement after that. A 35-year-old healthy patient presented with sudden and painless vision loss of her right eye, after coughing. During the ophthalmologic evaluation, she had a pre-macular hemorrhage and no other abnormalities. Initially, we chose for expectant management, but the hemorrhage did not clear totally. Thus, pars plana vitrectomy associated with internal limiting membrane peeling was indicated, with considerable improvement of her visual acuity, without perioperative complications or significant findings in the optical coherence tomography, autofluorescence and multifocal electroretinogram. In this case, sub-internal limiting membrane hemorrhage treatment with vitreoretinal surgery was relatively useful, with visual acuity improvement and resolution of sub-internal limiting membrane hemorrhage.
\end{abstract}

Keywords: Retinal hemorrhage; Valsalva maneuver; Vitrectomy; Tomography, optical coherence; Visual acuity; Humans; Male; Adult; Case report

\section{INTRODUÇÃO}

As hemorragias pré-retinianas geralmente ocorrem na interface entre a hialoide posterior e a membrana limitante interna (MLI). Menos frequentemente, elas estão localizadas na retina superficial, entre a MLI e a camada de fibras nervosas. Sua localização preferencial é na região macular levando, por conseguinte, à redução acentuada da acuidade visual|(1).

As hemorragias subMLI estão associadas a inúmeras causas, sendo a síndrome de Terson ${ }^{(2)}$ a mais comum. Outras possíveis causas são: discrasias sanguíneas ${ }^{(3)}$, trauma contuso da face ${ }^{(3)}$ e ruptura de macroaneurismas ${ }^{(4)}$, além da retinopatia por Valsalva ${ }^{(4)}$.

A retinopatia por Valsalva geralmente, apresenta-se como uma hemorragia pré-macular circunscrita, porém sua localização anatômica exata, subMLI ou subialoide, é ainda controversa e difícil de ser definida através da oftalmoscopia.

O objetivo deste relato é descrever o caso de uma paciente com retinopatia por Valsalva que evoluiu com hemorragia subMLI e foi submetida à vitrectomia via pars plana, tendo tido melhora da acuidade visual e seguimento através da tomografia de coerência óptica (OCT) de domínio espectral, além de ter sido submetida à autofluorescência e eletrorretinograma multifocal (mfERG).

\section{RELATO DE CASO}

Paciente do sexo feminino, 35 anos, sem comorbidades, procura o Pronto-Socorro de Oftalmologia com redução súbita e indolor da acuidade visual do olho direito (OD), após ter tido crise de tosse de forte intensidade, há um dia. Ao exame oftalmológico, apresentava acuidade visual com correção de conta dedos a $20 \mathrm{~cm}$ (OD) e 1,0 (olho esquerdo-OE). Apresentava motilidade ocular e reflexos preservados, pressão intraocular de $12 \mathrm{mmHg}$ em ambos os olhos (AO), sem alterações à biomicroscopia de $\mathrm{AO}$ ou à fundoscopia do OE. No OD, apresentava nervo óptico corado, retina aplicada e hemorragia pré-macular (Figura 1).

Realizou-se uma OCT Stratus ${ }^{\oplus}$, que mostrou-se inconclusiva quanto à localização exata da hemorragia.

Após explicações das possíveis condutas a serem tomadas, bem como seus riscos e benefícios, e por opção da paciente, optou-se por conduta expectante, havendo reabsorção parcial da hemorragia ao longo de dois meses (Figura 1), porém sem resolução total do quadro ou melhora da acuidade visual (conta dedos a 1,5 m). A fundoscopia do OD apresentava hemorragia de aspecto seroso. Diante disso, optou-se pela realização de vitrectomia via pars plana (material 23 gauge), com descolamento da hialoide posterior e observação da
Submetido para publicação: 14 de maio de 2012

Aceito para publicação: 12 de novembro de 2012

Trabalho realizado junto à Clínica Oftalmológica do Hospital das Clínicas da Faculdade de Medicina da Universidade de São Paulo - USP - São Paulo (SP) - Brasil.

Médico, Clínica Oftalmológica, Hospital das Clínicas, Faculdade de Medicina, Universidade de São Paulo - USP - São Paulo (SP) - Brasil.

${ }^{2}$ Médico, Setor de Retina e Vítreo, Clínica Oftalmológica, Hospital das Clínicas, Faculdade de Medicina, Universidade de São Paulo - USP - São Paulo (SP) - Brasil.
Financiamento: Não houve financiamento para este trabalho.

Divulgação de potenciais Conflitos de Interesse: M.M.Lavezzo, Nenhum; L.C.Zacharias, Nenhum, W.Y.Takahashi, Nenhum.

Endereço para correspondência: Marcelo Mendes Lavezzo. Rua Capote Valente, 136 - Apto. 54 São Paulo - (SP) 05409-000 - Brasil - E-mail: mmlavezzo@gmail.com 
manutenção do líquido de aspecto seroso. Diante disso, optou-se pelo "peeling" de MLI, utilizando pinça apropriada (Eckardt), com auxílio do corante azul brilhante, injetado com uma cânula "soft tip", permitindo a aspiração do líquido em questão através de uma pinça "backflush". Assim, concluiu-se de que se tratava de hemorragia de localização subMLI. Não houve intercorrências peri-operatórias. A paciente foi informada, através de termo de consentimento livre e esclarecido, dos riscos do procedimento proposto e estava de acordo com a realização do mesmo.

$\mathrm{Na}$ evolução, a paciente apresentou melhora da acuidade visual (0,1 - um mês de pós-operatório), sem alterações do segmento anterior ou pressão intraocular (PIO), com resolução do quadro fundoscópico, apresentando mácula sem edema ou outras alterações (Figura 1).

Foi realizado, no segundo mês de pós-operatório, o OCT (Heidelberg Engineering ${ }^{\oplus}$, Heidelberg, Germany) com tecnologia spectral domain, que demonstrou depressão foveal preservada (Figura 2). Também foi submetida à autofluorescência (VISUCAM 500, Zeiss,

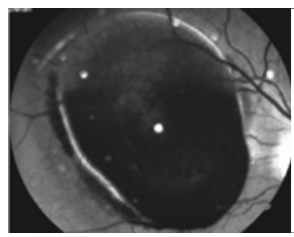

Julho/2011

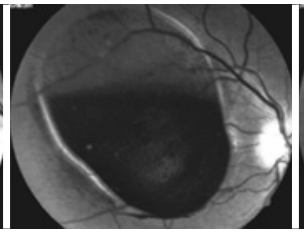

Agosto/2011

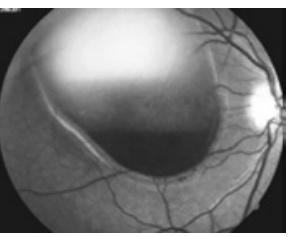

Setembro/2011

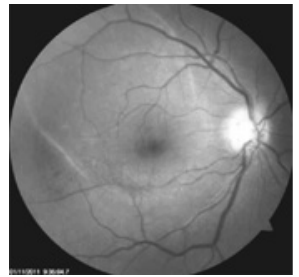

1 mês pós-operatório

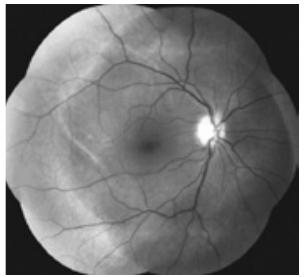

3ำ mês pós-operatório
Figura 1. Aspectos fundoscópicos do olho direito e sua evolução ao longo do tempo. Observa-se hemorragia pré-macular com reabsorção parcial em dois meses (aspecto seroso); pós-operatórios com resolução do quadro, com mácula dentro dos limites da normalidade.
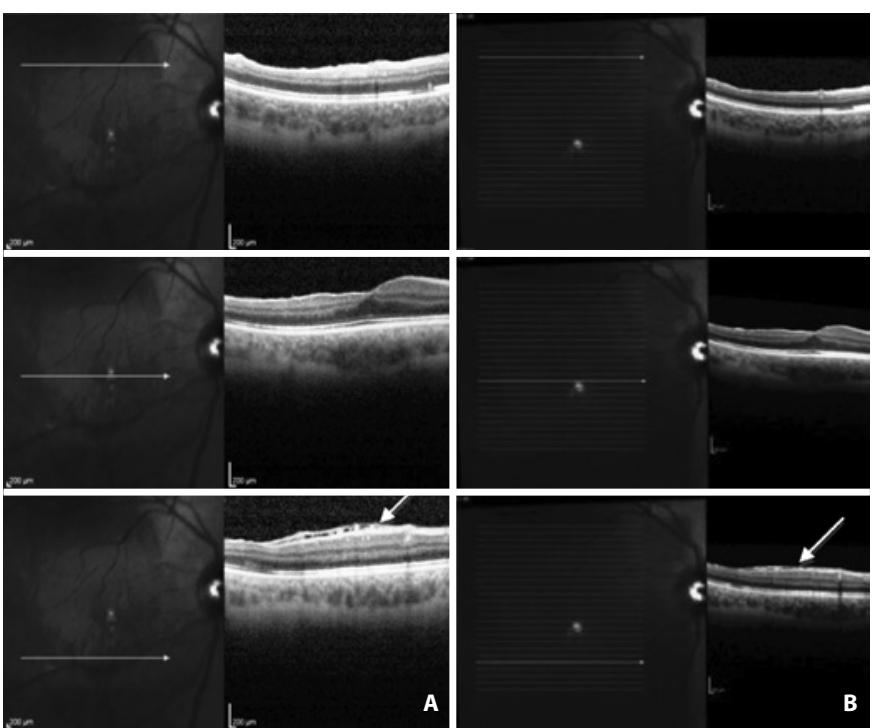

Figura 2. Tomografia de coerência óptica de alta resolução (OCT spectral domain) do olho direito revelando depressão foveal preservada e interface entre a região onde fo feito o "peeling" da MLI (setas brancas) (2 meses de pós-operatório (A) e 4 meses de pósoperatório (B). As espessuras centrais mínimas aferidas foram: $293 \mu \mathrm{m}$ (A) e $295 \mu \mathrm{m}$ (B). Observa-se que a camada de fotorreceptores apresenta-se íntegra.
Oberkochen, Germany), sendo visível a transição entre a região onde foi realizado o "peeling" de MLI e a região com MLI íntegra (Figura 3). O mfERG (RETIscan ${ }^{\circledR}$ multifocal ERG, ROLAND Instruments, Brandenburg, Germany) do OD não mostrou alterações significativas em relação ao OE (Figura 3).

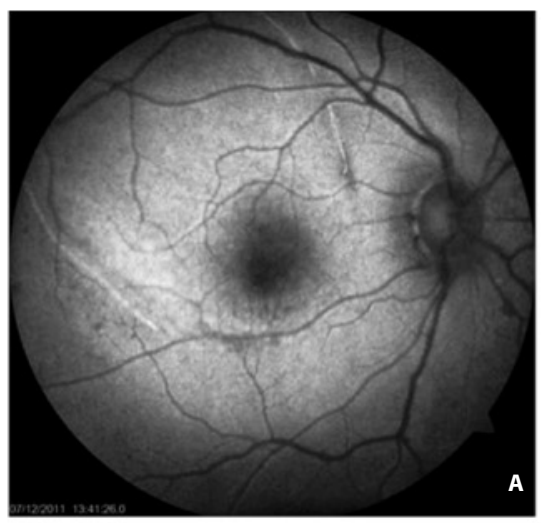

2o mês pós-operatório

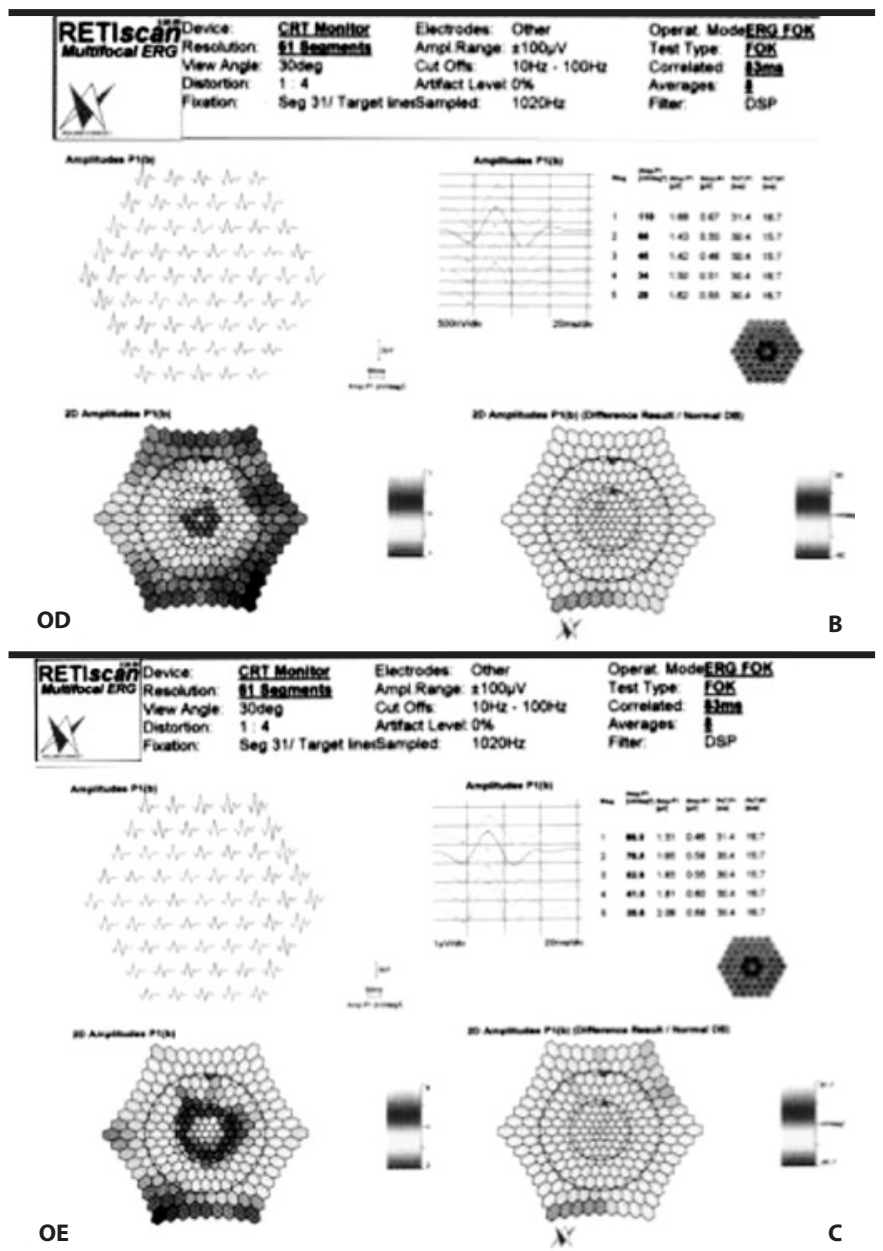

Figura 3. A) Autofluorescência do olho direito revelando a transição entre a região onde foi realizado o"peeling"de MLI e a região de MLI íntegra (linha hiperautofluorescente), sem outras alterações da autofluorescência da retina, no segundo mês de pós-operatório. Be C) Eletrorretinograma multifocal (mfERG) dentro dos limites da normalidade em ambos os olhos, com traçados de amplitude e latência dentro da normalidade e de aspecto relativamente uniforme nos vários pontos analisados (quinto mês de pós-operatório). 
Com seis meses de pós-operatório, a paciente encontra-se em seguimento ambulatorial, sem alterações substanciais do quadro, mantendo acuidade visual de 0,7, com melhor correção.

\section{DISCUSSÃO}

A retinopatia por Valsalva tipicamente se apresenta com uma redução súbita da acuidade visual em um indivíduo hígido, causada por uma hemorragia pré-macular secundária à manobra de Valsalva, cujas principais causas são: vômitos, tosse, esforço e atividades físi$\operatorname{cas}^{(5,6)}$. Há uma ruptura espontânea dos capilares perifoveais, devido ao aumento súbito da pressão venosa intraocular, secundária à elevação abrupta da pressão intratorácica ou intra-abdominal ${ }^{(7,8)}$.

As hemorragias subMLI aparecem fundoscopicamente como uma hemorragia bem demarcada. Entretanto, é frequentemente difícil distingui-la de uma hemorragia subialoidea. A OCT auxiliaria nesta diferenciação, porém, apesar da sua alta resolução, a confusão diagnóstica do nível em que se encontra a hemorragia pode ocorrer devido à alta refletividade do sangue em hemorragias recentes. Assim, o único método para confirmar a localização subMLI da hemorragia é a coloração intraoperatória da membrana sobre a hemorragia e, em alguns casos, o exame histopatológico da MLI(3). Este exame não foi realizado no presente caso, porém o aspecto cirúrgico da retirada da membrana limitante interna, bem como a interface da região do "peeling" evidenciada no OCT auxiliam na confirmação da retirada da MLI (Figura 2).

O nível histológico da hemorragia reflete o prognóstico visual em casos de ruptura de macroaneurismas e hemorragias maculares. Existe maior recuperação da acuidade visual em olhos nos quais havia hemorragia vítrea ou subMLI, do que quando há hemorragia submacular ${ }^{(4)}$.

Diante de um caso de retinopatia por Valsalva, geralmente, opta-se por uma conduta conservadora. Quase todos os pacientes apresentam uma recuperação visual favorável, à medida que a hemorragia é reabsorvida. No entanto, esta reabsorção pode ser muito lenta. $\mathrm{O}$ contato prolongado da retina com a hemoglobina e seus catabólitos pode causar dano retiniano tóxico irreversível. Outras complicações potenciais são: catarata, membrana epirretiniana, glaucoma, descolamento de retina, vitreorretinopatia proliferativa e ambliopia/miopia em crianças $^{(3)}$.

Vários estudos investigaram o uso do YAG laser para romper a MLI e drenar o sangue pré-retiniano em direção ao vítreo. Trata-se de um procedimento efetivo, levando à rápida recuperação visual, evitando, dessa forma, uma cirurgia vitreorretiniana. Todavia, casos de buraco macular, membrana epirretiniana e descolamento de retina tracional após YAG laser foram descritos na literatura ${ }^{(9,10)}$. Membranotomia com laser de argônio e de criptônio também já foram testados com algum sucesso. Outras modalidades incluem injeção intravítrea de gás (com ou sem t-PA), mobilizando a hemorragia da região foveal ${ }^{(3)}$.

O tratamento com a vitrectomia posterior mostrou-se efetivo, com melhora da acuidade visual significativa e imediata, prevenindo, inclusive, complicações relacionadas à presença de hemorragias intraoculares prolongadas. Assim, diante do exposto, optou-se pela realização da cirurgia vítreorretiniana, devido à não-absorção completa da hemorragia ao longo de três meses, bem como devido à sua localização subMLI, evitando a recuperação visual. As complicações mais comuns da vitrectomia são: aumento da pressão intraocular, catarata e descolamento de retina, nenhuma das quais foi observada no presente caso.

Há poucos casos na literatura de pacientes com retinopatia por Valsalva que se apresentavam com hemorragia subMLI, havendo evolução favorável na maioria deles. Em um caso de homem de 20 anos de idade, houve melhora da acuidade visual para 20/20, após a vitrectomia posterior. Em outro caso de um construtor de 24 anos de idade, houve melhora da acuidade visual de movimento de mãos para 20/20, após o tratamento(3). Além disso, não foram encontrados relatos na revisão bibliográfica realizada de casos de retinopatia por Valsalva submetidos à vitrectomia posterior e à avaliação funcional dos fotorreceptores através de ERG multifocal, que evidenciou de forma objetiva a integridade funcional dos fotorreceptores da paciente em questão, mesmo após 2 meses de evolução com a hemorragia pré-macular. Desse modo, pode-se afirmar que nem sempre ocorre a toxicidade da hemoglobina à camada de fotorreceptores já descrita em outras situações de hemorragia pré-macular ${ }^{(3)}$.

Desse modo, conclui-se que, diante de casos com hemorragia subMLI pós-Valsalva por tempo prolongado, causando redução importante da acuidade visual, a vitrectomia posterior com remoção da MLI pode ser uma opção segura e eficaz. Vale ressaltar, entretanto, que se trata de relato limitado a um paciente e houve seguimento por um curto período. Mais estudos devem ser realizados, com maior número de pacientes e seguimento mais prolongado, para se determinar o real benefício e o momento mais correto de se realizar a vitrectomia posterior em casos de hemorragia subMLI pós-Valsalva.

\section{REFERÊNCIAS}

1. Foos RY. Vitreoretinal juncture; topographical variations. Invest Ophthalmol. 1972; 11(10):801-8.

2. McCarron MO, Alberts MJ, McCarron P. A systematic review of Terson's syndrome: frequency and prognosis after subarachnoid haemorrhage. J Neurol Neurosurg Psychiatry. 2004;75(3):491-3.

3. De Maeyer K, Van Ginderdeuren R, Postelmans L, Stalmans P, Van Calster J. Sub-inner limiting membrane haemorrhage: causes and treatment with vitrectomy. $\mathrm{Br} J \mathrm{Oph}$ thalmol. 2007; 91(7): 869-72. Comment in: Br J Ophthalmol. 2007;91(7):850-2.

4. Nakamura H, Hayakawa K, Sawaguchi S, Gaja T, Nagamine N, Medoruma K. Visual outcome after vitreous, sub-internal limiting membrane, and/or submacular hemorrhage removal associated with ruptured retinal arterial macroaneurysms. Graefes Arch Clin Exp Ophthalmol. 2008;246(5): 661-9.

5. Georgiou T, Pearce IA, Taylor RH. Valsalva retinopathy associated with blowing balloons. Eye (Lond). 1999;13( Pt 5):686-7.

6. Ladjimi A, Zaouali S, Messaoud R, Ben Yahia S, Attia S, Jenzri S, et al. Valsalva retinopathy induced by labour. Eur J Ophthalmol. 2002;12(4):336-8. Comment in: Eur J Ophthalmol. 2003;13(1):113.

7. Tatlipinar S, Shah SM, Nguyen QD. Optical coherence tomography features of sub-internal limiting membrane hemorrhage and preretinal membrane in Valsalva retinopathy. Can J Ophthalmol. 2007;42(1):129-30.

8. Meyer CH, Mennel S, Rodrigues EB, Schmidt JC. Persistent premacular cavity after membranotomy in valsalva retinopathy evident by optical coherence tomography. Retina. 2006;26(1):116-8.

9. Durukan AH, Kerimoglu H, Erdurman C, Demirel A, Karagul S. Long-term results of $\mathrm{Nd}$ :YAG laser treatment for premacular subhyaloid haemorrhage owing to Valsalva retinopathy. Eye (Lond). 2008;22(2):214-8.

10. Aralikatti AK, Haridas AS, Smith JM. Delayed Nd:YAG laser membranotomy for traumatic premacular hemorrhage. Arch Ophthalmol. 2006;124(10):1503. 